\title{
e-Phaïstos
}

e-Phaïstos

Revue d'histoire des techniques / Journal of the history

of technology

VI-1 2017 | 2018

Varia

\section{L'espace conservatoire des îles Kerkenna et la mouvance temporelle de traditions nautiques}

The conservative space of the Kerkennah Islands and the evolution of the nautical traditions

\section{Patrick FÉRON}

\section{(2) OpenEdition}

Journals

Édition électronique

URL : http://journals.openedition.org/ephaistos/3028

DOI : 10.4000/ephaistos.3028

ISSN : 2552-0741

Éditeur

IHMC - Institut d'histoire moderne et contemporaine (UMR 8066)

Référence électronique

Patrick FÉRON, «L'espace conservatoire des îles Kerkenna et la mouvance temporelle de traditions nautiques », e-Phaïstos [En ligne], VI-1 2017 | 2018, mis en ligne le 30 juin 2018, consulté le 22 avril 2019. URL : http://journals.openedition.org/ephaistos/3028 ; DOI : 10.4000/ephaistos.3028

Ce document a été généré automatiquement le 22 avril 2019

Tous droits réservés 


\section{L'espace conservatoire des îles Kerkenna et la mouvance temporelle de traditions nautiques}

The conservative space of the Kerkennah Islands and the evolution of the nautical traditions

Patrick Féron

\section{Introduction}

1 La richesse naturelle, culturelle et technique concentrée dans l'espace nautique tunisien, fait de ce «microcosme» un support de référence peu commun. Les caractéristiques hydrographique, hydrologique et halieutique de cet espace resserré, se mesurent à l'échelle de la partie orientale méditerranéenne. Le profil du golfe de Gabès où siègent les îles Kerkenna, permet de matérialiser des eaux peu profondes, des familles de bateaux de travail, des métissages originaux, des mobilités nautiques et des modes d'exploitation vivriers ou de transport. Ces îles s'intégraient à la " Petite Syrte », elles tissaient des liens avec le littoral tunisien, elles communiquaient avec Malte, les côtes grecques et la mer Adriatique. Se dessinent alors les marges extrêmes de leur positionnement dans la partie orientale de la Méditerranée. Dans ce contexte spatial, les principes de construction vernaculaires pleinement inféodés au milieu naturel témoignent d'évolutions contemporaines encore observables dans l'archipel des îles Kerkenna.

2 L'essor de la population au Kerkenna est significatif car jusqu'au XVIII ${ }^{\mathrm{e}}$ siècle, par exemple, l'île Mellita est presque déserte ${ }^{1}$. Servonnet et Laffite exposent une perspective temporelle qui, à la fin du XIX ${ }^{e}$ siècle, dresse un premier état global des lieux. Lequel mesure l'incidence de la circulation des hommes, des savoirs et de l'appropriation de savoir-faire, à travers la présence saisonnière remarquable des pêcheurs d'éponges grecs, et à la lumière de l'évaluation d'une population d'origine maltaise sédentarisée, beaucoup plus versée dans le domaine maritime. Une fraction de Maltais monopolise le champ de la 
construction navale, ces charpentiers maîtrisent la fabrication de barques, où réside l'aïeule de la fluka $a^{2}$, une embarcation de pêche dotée d'une voile latine très répandue sur l'archipel aujourd'hui.

3 La mémoire de bateaux vernaculaires qui gravitent dans le golfe de Gabès apparaît très distinctement dans la littérature et l'iconographie vers la fin du XIX ${ }^{\mathrm{e}}$ siècle alors que l'ensemble du littoral tunisien ne propose encore que des abris naturels. Le lac de Bizerte, le lac de Tunis et la lagune de Djerba forment des espaces lacustres peu profonds. Le golfe de Gabès, mer étroite ou "narrow-sea » selon l'expression chère à Fernand Braudel ${ }^{3}$, est festonné d'un maillage de hauts fonds qui bordent le littoral. Ils encerclent l'archipel des Kerkenna d'un large plateau sous-marin que percent des canaux navigables. Une telle configuration ne manque pas d'influencer les principes de construction de menues embarcations, de bateaux de pêche, de caboteurs de commerce, propulsés à l'aviron, à la voile, se déplaçant dans un espace nautique multiforme.

4 De par ce panorama, le golfe de Gabès et l'archipel des Kerkenna suscitent une réflexion gouvernée par l'originalité d'un foyer historique et contemporain de traditions nautiques d'une densité remarquable. Le qualificatif « d'espace conservateur ${ }^{4}$ » que lui attribue Abdelhamid Barkaoui, à propos de la construction navale traditionnelle, accroît l'attrait patrimonial de ce bassin et renforce son pouvoir d'attraction. L'objet de la réflexion est d'extraire des clés de lecture et d'interprétation relatives à la mouvance temporelle de traditions d'architecture nautique dans ce milieu d'exception. Sollicité par l'attraction des îles Kerkenna nous exposerons au préalable les supports de réalisation de l'étude documentaire et de terrain. Cette mission de recherche a été réalisée dans le cadre du programme mobilité HERITECHS, coordonné par le centre d'histoire des techniques, IHMC, Paris 1 Panthéon-Sorbonne, en partenariat avec l'Université de Sfax, Tunisie.

\section{L'attraction des Kerkenna}

5 À l'ouest de la presqu'île du Cotentin, en Normandie, s'égraine un cortège d'îles et d'archipels français et anglo-normands ${ }^{5}$. Notre sensibilité à la notion d'espace nautique et d'insularité tient à la fréquentation de ce bassin de navigation parcouru en voilier. Un dédale archipelagique poissonneux, bordé au sud par la baie du Mont Saint Michel où siègent les plus fortes marées d'Europe. Ce bassin maritime est riche d'une culture nautique à cheval sur le monde anglo-normand, il est soumis à l'alternance de forts courants de marée et aux variations météorologiques de cette latitude.

6 Aussi, l'archipel des îles Kerkenna suscite-t-il une interrogation gouvernée par l'attrait d'un faciès océanographique archétypale, foyer de traditions culturelles et techniques remarquables. Il s'étend au large de Sfax, sur la bordure africaine du bassin oriental méditerranéen. La réflexion vise à explorer le concept " espace nautique conservateur " résultant de l'étude d'une barque de pêche locale: la fluka arbi ${ }^{6}$. Elle mobilise une approche historique, ethnographique, naturaliste et spatiale, en regard de facteurs de pérennité de cultures nautiques anciennes et contemporaines ou de sujets de disparitions, tel le loud ou loude qui fut le voilier polyvalent, de pêche et de transport, le plus représentatif. La fluka est une menue embarcation construite "sur quille " très répandue à Kerkenna aujourd'hui. Comparativement, le loude est un bateau plus grand, construit "à fond plat», il est doté d'un plan de voilure différent, autant de caractéristiques que nous examinerons dans le détail. 
7 L'étude ethnographique des îles Kerkenna réalisée par André Louis, entre 1956 et 1961, initiée dès 1945, donne une mesure de la richesse et de la diversité des activités humaines inféodées au milieu nautique kerkennien. Son recul de près de soixante-dix ans propose une somme de données de référence des plus utiles au chercheur. Ce travail fondateur, qui témoigne d'alliances et de convergences entre le milieu, l'homme et ses industries ${ }^{7}$, intervient à l'orée de changements irréversibles.

8 Notre entretien avec le professeur Abdelhamid Barkaoui ${ }^{8}$ a étayé notre perception générale de l'espace nautique des Kerkenna. Ses publications ${ }^{9}$, les travaux de Lucien Basch $^{10}$ et d'autres spécialistes rassemblés dans les Actes de la table ronde internationale, tenue à Sfax, en $2005^{11}$, confirment l'intérêt scientifique de l'iconographie. Nous avions constaté qu'au-delà des textes où s'affichent différentes traditions d'architecture nautique et qui permettent de situer leur usage, l'iconographie s'impose comme un outil incontournable qui comble l'insuffisance descriptive de l'écrit. D'où l'importance d'identifier graphiquement les "objets flottants» chaque fois que possible afin de faciliter leur compréhension. Ce mode d'investigation puise aux registres les plus divers, manuscrits, ouvrages imprimés, fonds iconographiques et photos contemporaines. Sur le terrain insulaire et maritime de l'archipel kerkennien, s'ajoute l'observation des collections conservées au Musée-résidence El Ferhi à Abassia et commentées par monsieur Abdelhamid Ferhi, créateur et conservateur du musée. Lors de la visite du chantier naval situé à l'Attaya, monsieur Sta Zohir Chelli, charpentier de marine ${ }^{12}$ nous a initiés à son art, confirmant l'abandon du bois d'olivier, devenu plus rare et trop coûteux, au profit de l'eucalyptus pour réaliser l'ossature des coques. Ce parcours nous a permis de détailler un unique exemplaire connu à ce jour, du loude d'el Khraïb-Beït-en-Najet, conservé sous un $\mathrm{abri}^{13}$. Cette tradition d'architecture nautique renvoie à l'ouvrage de Servonnet et Laffite $^{14}$, d'Hennique ${ }^{15}$ et aux travaux de Gateau ${ }^{16}$ qui nous rappellent combien était grande la diversité des embarcations fréquentant l'archipel des Kerkenna et la partie orientale de la Méditerranée.

9 L'océanographie, les ressources naturelles, les modes d'exploitation, les moyens de navigation organisent les lignes de force mobilisées pour l'exploration culturelle et technique des Kerkenna et l'appréhender sous l'angle de la notion «espace nautique conservateur ».

10 L'espace terrestre insulaire, sa préemption du domaine maritime, converti en droits, la régénération naturelle du vivier halieutique, les bois et végétaux de construction, les traditions d'architecture nautique, la navigation à voile, les savoirs et les savoir-faire, composent une somme de particularismes que protège encore partiellement le microcosme méditerranéen d'un archipel ancré à quelques encablures de Sfax.

\section{Trois clés d'interprétation}

\section{La corrélation entre la marée et l'hydrographie}

11 Fernand Braudel décrit la Méditerranée comme «une succession de plaines liquides communiquant entre elles par des portes plus ou moins larges ${ }^{17} »$. Il individualise les bassins Ouest et Est, en " une série de mers étroites, de narrow-seas » où se développent des caractères, des types de bateaux, des usages, des "lois propres d'histoire", considérant que parmi ces « mondes » fractionnés, les plus riches sont les plus étroits. Le golfe de Gabès entre sans nul doute dans cette catégorie et, dans sa partie nord, l'archipel 
des Kerkenna, posé sur un plateau de hauts fonds, décline un microcosme encore plus singulier. Il est le foyer de traditions influencées par des facteurs naturels remarquables que sont l'amplitude de la marée, une permanence de zones de calme et de grandes ressources halieutiques. Ces éléments naturels ont promu le développement de traditions maritimes vernaculaires et entretiennent le reliquat que l'on observe aujourd'hui.

Selon la bibliographie, jusqu'à l'arrivée du moteur auxiliaire, l'accès aux Kerkenna était réputé difficile pour les navires étrangers à cause de hauts fonds et de la forte marée. Le superlatif qualifiant la marée de cette zone méditerranéenne ne manquait de surprendre comparativement à la baie du Mont Saint Michel, siège remarquable des plus fortes marées d'Europe auxquelles nous sommes accoutumés. Aussi, l'évaluation du marnage observé dans l'archipel kerkennien, joint à l'emprise du faciès hydrographique creusé de chenaux navigables, sollicitait-il une interrogation.

Une consultation documentaire nous confirmait la valeur relative du terme « forte marée 18 ". Localement, la région du golfe de Gabès, où pénètre une boucle du courant général de la Méditerranée, présente la plus grande amplitude de marée. Comparativement, l'Adriatique bénéficie d'un marnage moindre, son amplitude dépasse rarement 1 mètre. Le niveau maximum en mer Ionienne approche rarement 0,75 mètre; à Tripoli et dans le golfe de Syrte, les oscillations dues au vent atteignent 0,60 mètre ${ }^{19}$. À Gabès, l'amplitude dépasse 2 mètres, selon l'océanologue tunisien Sammar Chérif « ce marnage inhabituel en Méditerranée, non entièrement expliqué, est dû à un phénomène de résonance que rencontre l'onde principale de marée dans le bassin compris entre le Ras Kapudia et Zrazis $^{20} »$.

14 Cette élévation du niveau de la mer, unique en Méditerranée, parait bien modeste comparée à l'amplitude de 14,50 mètres relevée en baie du mont Saint-Michel. Mais l'incidence de 2 mètres de marnage se manifestant sur les faibles profondeurs de Kerkenna provoque une variation relative considérable. Dans les deux cas le brassage biquotidien des eaux favorise la richesse halieutique du milieu marin. Parmi les quatre éléments naturels remarquables qui caractérisent le golfe de Gabès, on note donc l'amplitude de la marée ; de faibles profondeurs, des zones de calme par presque tous les temps, et la richesse des ressources biologiques marines et végétales, piliers de la conservation de traditions que l'on observe encore aujourd'hui à Kerkenna.

\section{Un pré carré poissonneux}

15 La pêche aux îles Kerkenna ${ }^{21}$ se distingue par une entorse au principe du domaine public maritime communément admis, dans la mesure où l'activité halieutique relève de titres de propriété vieux de deux ou trois siècles. Ainsi, les pêcheries représentent-elles une véritable extension du domaine privé insulaire. Des droits authentifiés au XIX ${ }^{\mathrm{e}}$ siècle par des actes officiels sont conservés encore de nos jours par certaines familles kerkenniennes.

L'origine de cette appropriation remonterait au XVIII ${ }^{e}$ siècle sur l'initiative de deux sfaxiens dénommés Charfi qui interdirent aux Kerkenniens l'implantation de pêcheries sur le banc de la Taâdiya qui déborde la côte sud-ouest de l'île Mellita. Les villageois protestèrent et le Bey Ali attribua l'exploitation du banc aux pauvres et aux nécessiteux sous forme de donation aumônière. Au fil de la gestion des différents entre villages, de réclamations au nom du droit coutumier avancées par certaines familles et donnant lieu à des actes notariés, on s'achemina vers une formalisation du droit de propriété. L'ardeur 
des Kerkenniens à défendre leurs droits sur le domaine maritime périphérique s'explique par la richesse halieutique du milieu, ce don de la nature résulte de la conjonction entre une faible profondeur favorisant le rayonnement solaire et le maintient d'une bonne température et d'autre part, la circulation alternée des courants de marée qui véhiculent les éléments nutritifs favorables au renouvellement de la flore sous-marine et à la production du plancton.

Le commensalisme entre les insulaires et l'anneau de hauts fonds prolifiques, relève de ce faciès hydrographique singulier qui ceinture l'île et fonde l'ancrage d'une tradition halieutique ancestrale (fig.1). Le vaste plateau à fleur d'eau entretien la durabilité d'un "pré carré poissonneux » où règne aujourd'hui la fluka qui a succédé au loude. Cette petite embarcation à voile latine, plébiscitée par les pêcheurs pour sa fonctionnalité est particulièrement marine grâce à sa construction sur quille. La réduction extrême de son tirant d'eau l'inscrit en congruence avec le plateau hydrographique qui porte l'archipel. (fig.2, 3, 4)

L'archipel des Kerkenna, Tunisie.

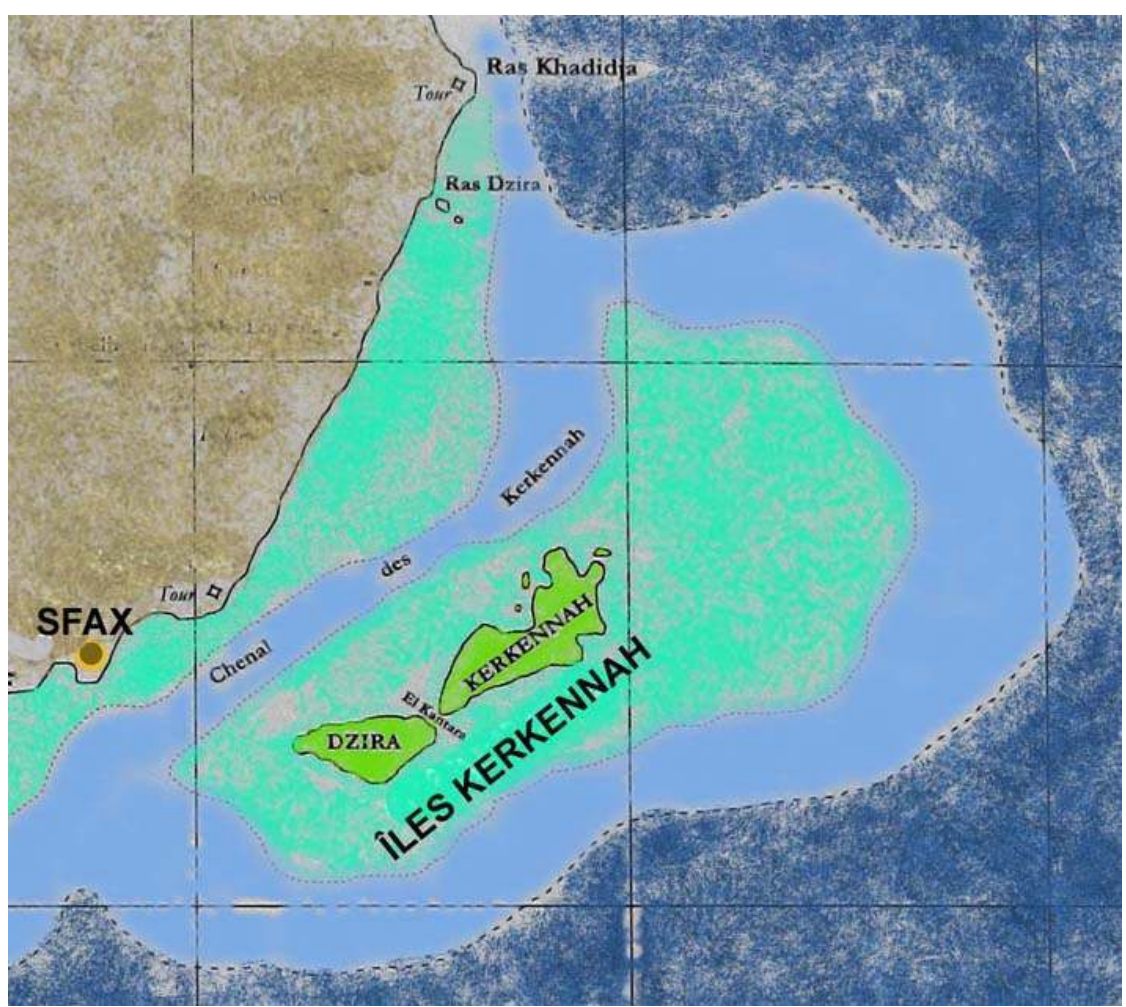

Le plateau peu profond a favorisé l'installation des pêcheries fixes.

P. Feron 2014 
Fluka : une construction sur quille, à plate-varangue, chantier de l'Attaya, Kerkenna

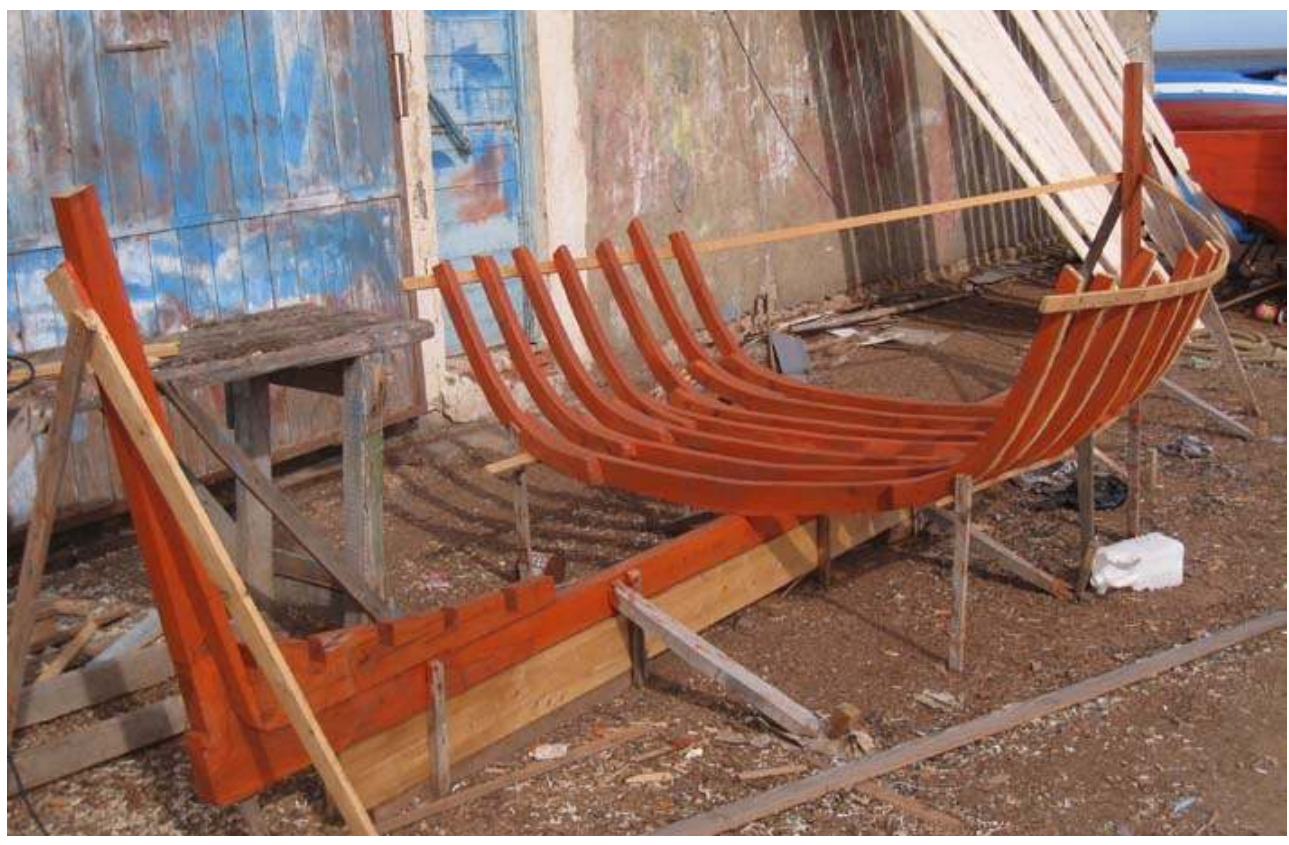

P. Feron 2014

\section{Fluka échouée à marée basse sur le rivage d'Abassia, Kerkenna}

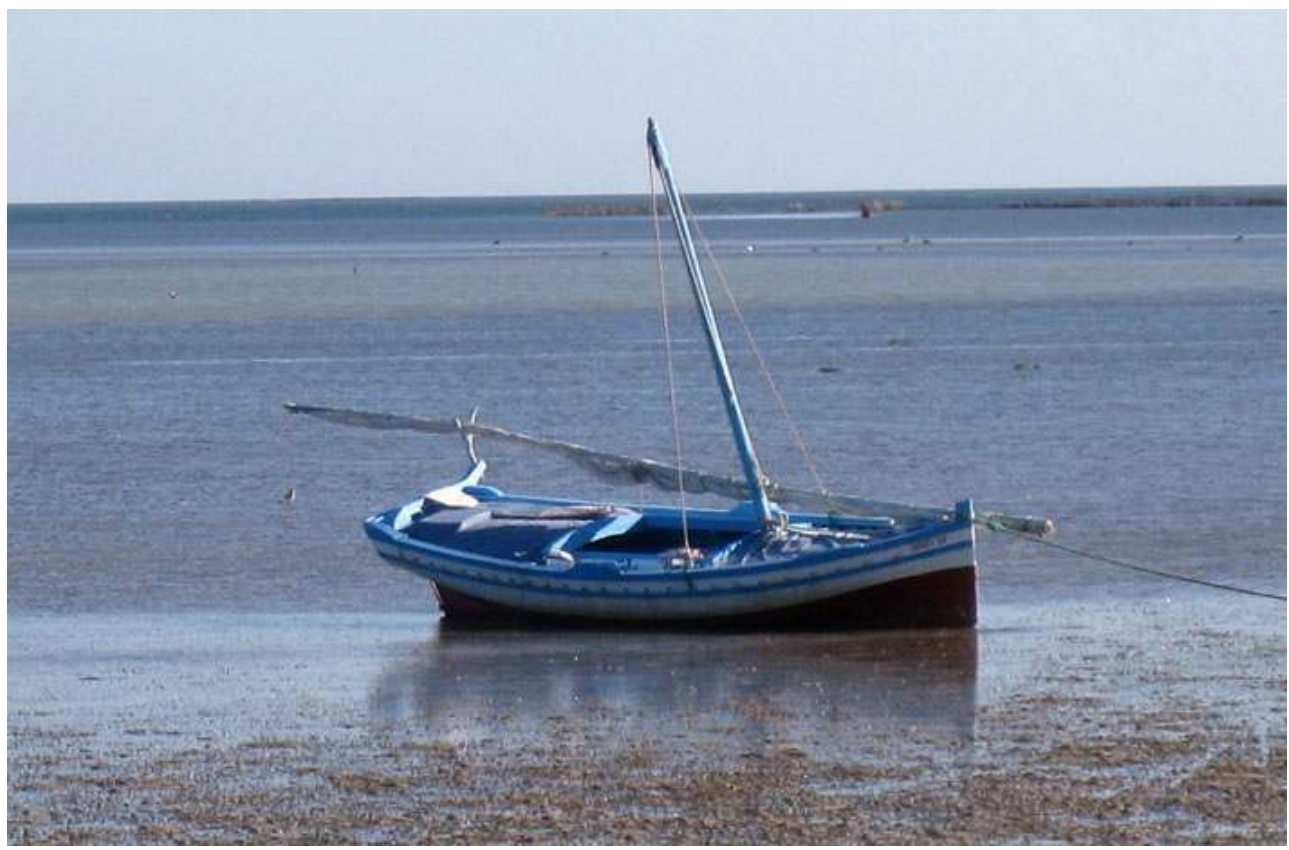

P. Feron 2014 


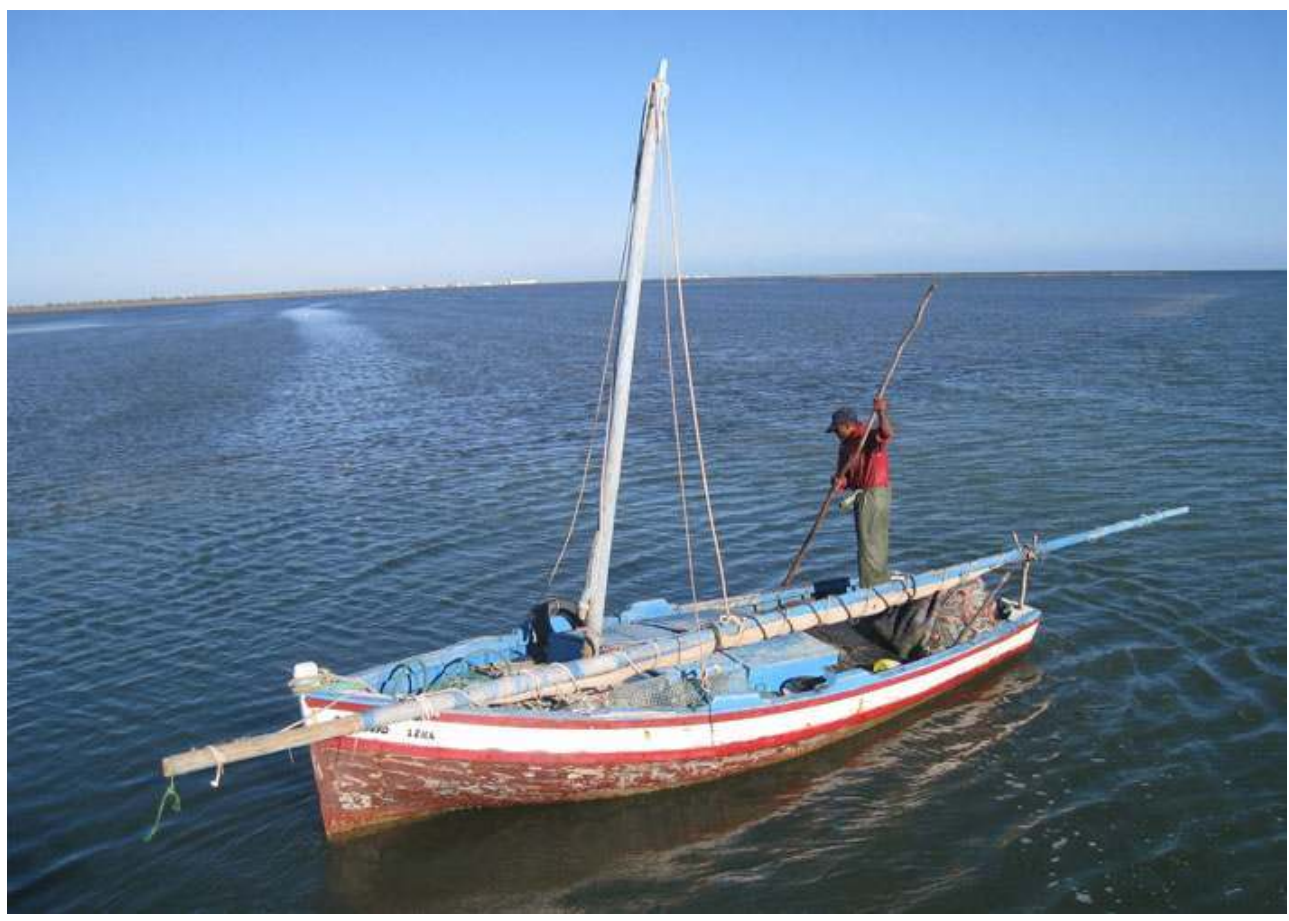

P. Feron, 2014

\section{Le triptyque « hauts fonds, palmier \& savoir-faire »}

L'expansion de l'exploitation des bancs du large où s'opèrent le piégeage du poisson et la capture du poulpe tient au palmier contributeur d'un système technique de capture très élaboré. Le mode de confection traditionnel des pêcheries instauré par les insulaires de Kerkenna provient de la transformation ingénieuse de cette ressource végétale terrestre. Les palmes du palmier récoltées et spécialement préparées, sont fichées sur le support stable des hauts fonds où elles se jouxtent et forment un barrage linéaire perméable aux courants.

Le palmier intervient jusque dans les moindres détails dans la confection traditionnelle d'une pêcherie fixe. L'implantation du chemin de capture consomme de 2500 à 4000 palmes qui sont enfoncées dans le sol sédimentaire, «Plus les chemins de capture et barrières de capture sont longs et nombreux (...), plus la zone de prise est importante et plus le produit de la pêche risque d'être fructueux ${ }^{22}$ » (fig.5, 6). L'opération se poursuit par le montage de claies, elles sont maintenues par des piquets pour constituer la chambre de capture. Les nasses réalisées en régimes de palmier étaient assujetties aux chambres à l'aide de cordelettes de sparte ${ }^{23}$.

20 Le palmier, pourvoyeur inépuisable de palmes, offre une production peu coûteuse qui alimente les besoins d'une technique de capture très consommatrice en matériaux. Sa prodigalité concourt à pérenniser la pêcherie fixe, l'arbre s'impose comme un facteur végétal de résilience face aux produits synthétiques coûteux. Intégrée dans ce complexe technique de capture du poisson, la fluka, outil de prédilection du pêcheur insulaire, apparait aujourd'hui comme le marqueur d'une tradition d'architecture nautique qui résiste à la modernisation des techniques de pêche que l'on observe néanmoins aux 
Kerkenna comme partout ailleurs. Cette description sommaire illustre le triptyque " hauts fonds, palmier \& savoir-faire », fondement de la tradition de la pêcherie fixe.

\section{Houppier de palmier.}

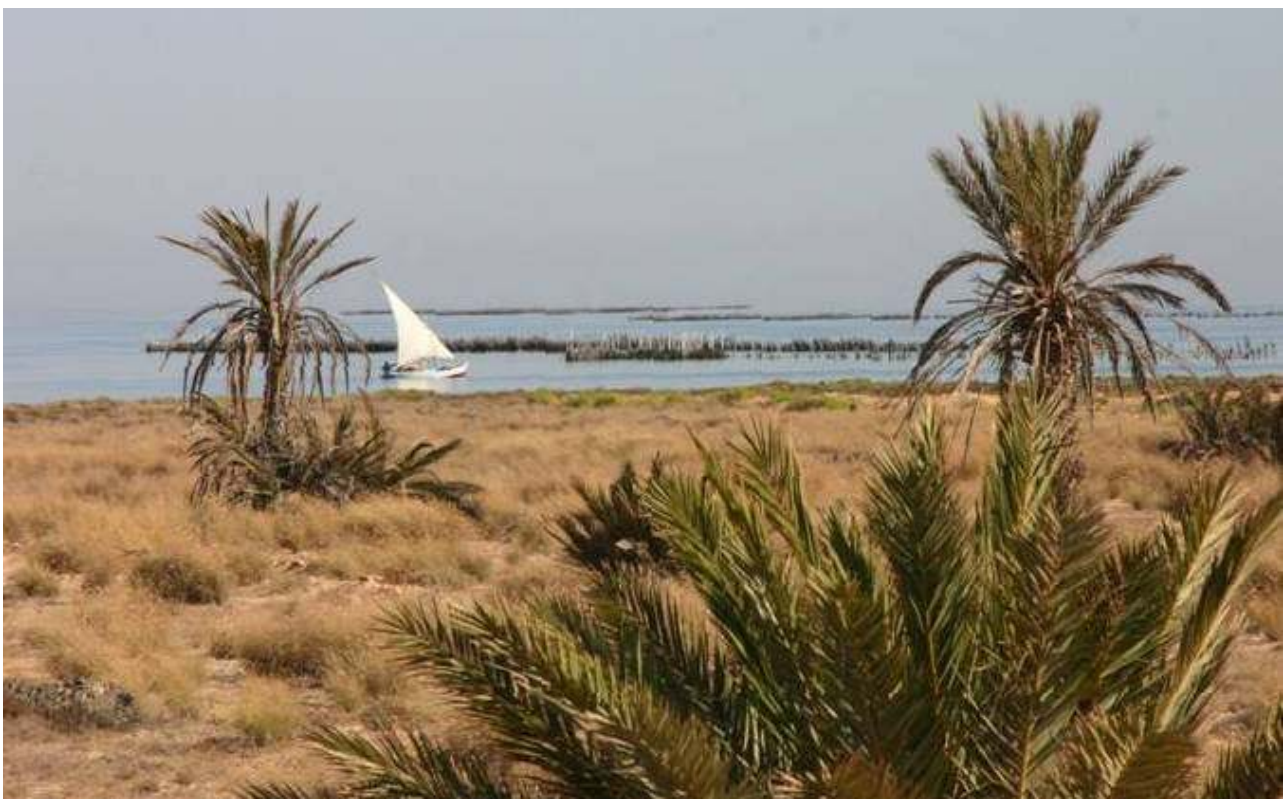

Les palmes servent à réaliser les chemins de capture.

Photo Wikipedia

Pêcherie fixe adossée au rivage.

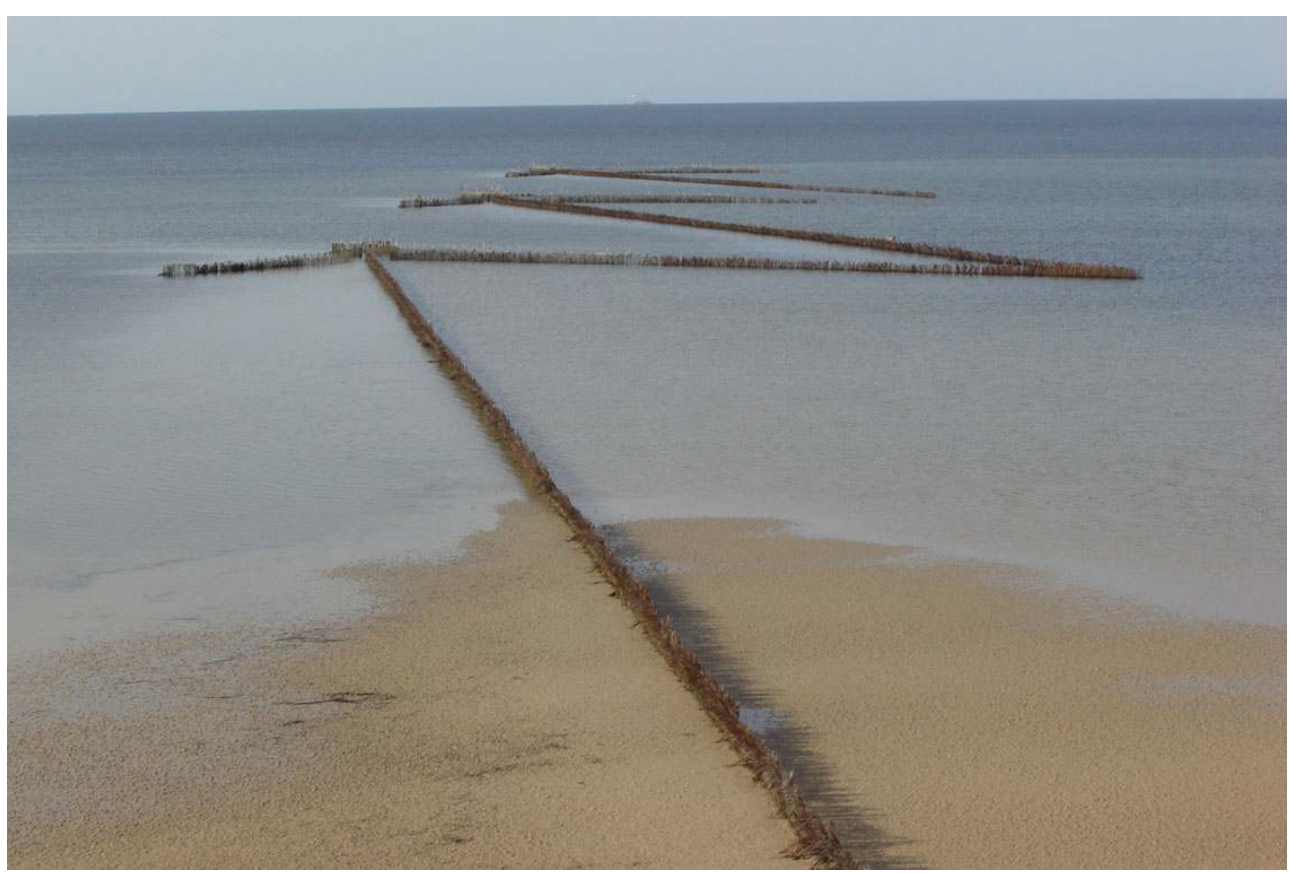

Photo Wikipedia. 


\section{La mouvance temporelle de traditions d'architecture nautique}

21 Nous avons évoqué la fluka, marqueur de traditions d'architecture nautique, André Louis nous livre un repère d'expansion de ce bateau en signalant que la construction de la felouque ou fluka se répand davantage sur la côte orientale des Kerkenna où plusieurs villages l'utilisent exclusivement ${ }^{24}$. De même nous introduit-il dans l'univers mémoriel de moyens nautiques vernaculaires disparus : l'etchem et le loude.

Fait rare en milieu maritime méditerranéen, l'image d'un etchem a été fixée par un photographe en $1925^{25}$, elle nous renvoie aux sources premières de la tradition monoxyle assemblé. Le radeau ou echtem, était confectionné avec trois ou quatre quartiers en stipe de palmier, d'une longueur de 2 à 3 mètres. Les éléments longitudinaux étaient assemblés entre-eux par des traverses de bois d'olivier insérées en force dans deux ou trois évidements perforant les stipes, elles dépassaient en largeur. Le radeau servait à la visite des pêcheries et à la pêche aux nasses. De vieux marins pratiquaient encore la pêche «à nasses libres » en radeau, de la Branca à la pointe de Sidi Founkhal, de Sidi Founkhal à Ber. La propulsion s'effectuait à la gaffe ou à la perche ${ }^{26}$ campé sur ses deux jambes écartées, le pêcheur portait devant lui un lot de nasses et de paniers à poissons. Il se déplaçait dans les sillons tracés dans les champs d'algues ou par les passages ouverts dans les ruines romaines de l'antique Cercinna, y déposant la nasse lestée ; au jusant le poisson guidé par les couloirs de pierre s'emprisonnait dans la nasse. Le radeau permettait d'aborder des zones rocheuses agressives pour le fond des barques, dans les endroits profonds le risque de chute rendait son usage dangereux ${ }^{27}$. En 1882, Hennique signale encore l'usage du echtem pour rejoindre les barques et gagner les plateaux éloignés du rivage. Le pêcheur que nous avons rencontré à Kellabine en 2014, se servait d'une planche à voile pour transporter le moteur hors-bord jusqu'à sa fluka.

23 André Louis aborde les Kerkenna pour la première fois en 1945, à bord d'un loude, il en livre le portrait de son époque. Le bateau était affecté au transport et à la pêche, sa coque mesurait communément 11 mètres de longueur. Son tirant d'eau était de 0,50 mètre, et sa capacité moyenne de 5 tonneaux. Son architecture et son gréement, le classe comme le plus petit échantillon de la famille des carèbes ${ }^{28}$. Évaluée à environ une centaine d'unités, en 1882, la flottille de carèbes se répartissait majoritairement entre Sfax, Kerkenna et Djerba. Elle était parfaitement adaptée au cabotage entre Sousse et Tripoli, serrant la côte au plus près, précise Hennique. La construction très originale du carèbe est l'œuvre des charpentiers tunisiens de cette côte. Comme toutes les barques arabes de cette région, l'ossature, les pièces de liaison sont en olivier, un bois réputé pour sa solidité, les bordages et les autres parties sont en sapin, de ce fait il apparaît que l'eucalyptus employé à l'heure actuelle, est encore inusité ${ }^{2}$.

Émanation des plus petits carèbes avec lesquels leur construction et leur gréement se confondent, les loudes à un ou deux mâts opèrent principalement sur les plateaux qui auréolent l'archipel des Kerkenna, Sfax et la mer intérieure de Djerba. L'identification de ce bel exemple d'hybridation lignagère échappe à tous les dictionnaires consultés par le commandant Hennique ${ }^{30}$ en 1882. Le second portrait se traduit ostensiblement par un grand mât implanté sur l'avant du milieu, la voile trapézoïdale à bourcet est hissée à son sommet, l'inclinaison remarquable de l'espar présente une forte quête sur l'arrière contrôlée par un étai réglable à palan. Par petit temps, le loude grée une petite voile 
triangulaire à bordure libre transfilée sur une perche amovible, courte et flexible, "plantée » dans un trou d'étambrai situé à l'extrême avant. Sous voilure complète la gémellité avec le carèbe tunisien est frappante, son entrée d'eau et la façon arrière sont fines, sa muraille peu élevée s'accorde avec un faible tirant d'eau; quand la brise forcit, le triangle de toile avant est facilement rentré en sortant le mât non haubané de l'étambrai. La grande voile garnie de 6 à 8 bandes de ris voit sa surface réduite de moitié si nécessaire, cette faculté permet de prolonger la navigation sur les plateaux abrités. Ainsi voyait-on les loudes poursuivre le relevage des nasses des pêcheries de la rade par vent mauvais; ils assuraient, pour l'essentiel, les liaisons entre Sfax et les Kerkenna, et fréquentaient abondamment la mer intérieure de Djerba pendant la saison de la pêche des éponges.

Ultime représentant d'une lignée génératrice de métissages techniques issus des carèbes, le loude d'el-Kraïb s'affiche tel un passeur de mémoire grandeur nature. Pour plus de détails on se reportera à l'examen réalisé par Abdelhamid Barkaoui il y a une douzaine d'années ${ }^{31}$. Le qualificatif de "barque à fond plat $"^{32}$ figurant dans certains textes commandait l'examen visuel de ce modèle de référence. Malgré son conditionnement sous un abri, le bateau a souffert de l'usure du temps et présente de fâcheuses dégradations. Son état de vétusté n'autorisait qu'une expertise prudente. Il apparaît clairement que le fond du corps principal de la coque est composé de varangues plates recevant les bordages de fond (fig.7). Puis, les lignes d'eau des extrémités se resserrent harmonieusement en forme de $\mathrm{V}$ et aboutissent en pointe sur l'étrave et l'étambot (fig.8). Chacune de ces deux parties pincées, entrée et sortie d'eau, est nervurée à sa base par un court embryon structurant l'assemblage ossature et bordage, on le qualifie d'embryon de quille. Le bateau est présenté dans la configuration d'échouage sur la laisse de basse mer, circonstance courante qui, selon Hennique ${ }^{33}$, conduit le patron pêcheur à glisser une grosse pierre sous chaque embryon de quille afin de soustraire la coque au contact de la vase $^{34}$ (fig.9). Les fargues qui exhaussent le bordage sur la partie arrière sont encore partiellement en place, elles se raccordent au petit tableau triangulaire, fermant un demipont où se tenait le barreur (fig.10), la cale centrale, recevant les charges, reste ouverte. La section avant a perdu le pont qui courait du mât principal jusqu'à l'étrave, offrant une plate-forme de travail ergonomique en action de pêche ou pour la propulsion à la perche. Le dispositif d'étambrai traduit une grande robustesse, il se compose de deux forts barrots transversaux rapprochés recevant de part et d'autre de l'axe médian deux solides entremises (fig.11). Les entremises maintiennent l'espar latéralement, leur écartement réserve une lumière rectangulaire au passage du mât dont le pied vient reposer sur un sabot d'emplanture. L'intervalle d'une cinquantaine de centimètres séparant les barrots détermine l'angle d'inclinaison du mât sur l'arrière, comme le restituent de façon si caractéristique les gravures. La partie centrale du fond de la coque, dégarnie partiellement de ses bordages fournit un éclaté qui dévoile nettement les varangues plates, elles sont prolongées à chaque extrémité par une seule allonge de courbe dessinant un bouchain rond, avant de s'élever à la verticale vers le livet. La signature technique de cette «barque à fond plat » est originale, elle se démarque du fond plat à bouchain vif plus facilement identifiable, par la conception d'un bouchain rond plus complexe à réaliser en bois qui justifie la nervure structurante ou embryon de quille. 
Loude d'el Khraïb, Kerkennah

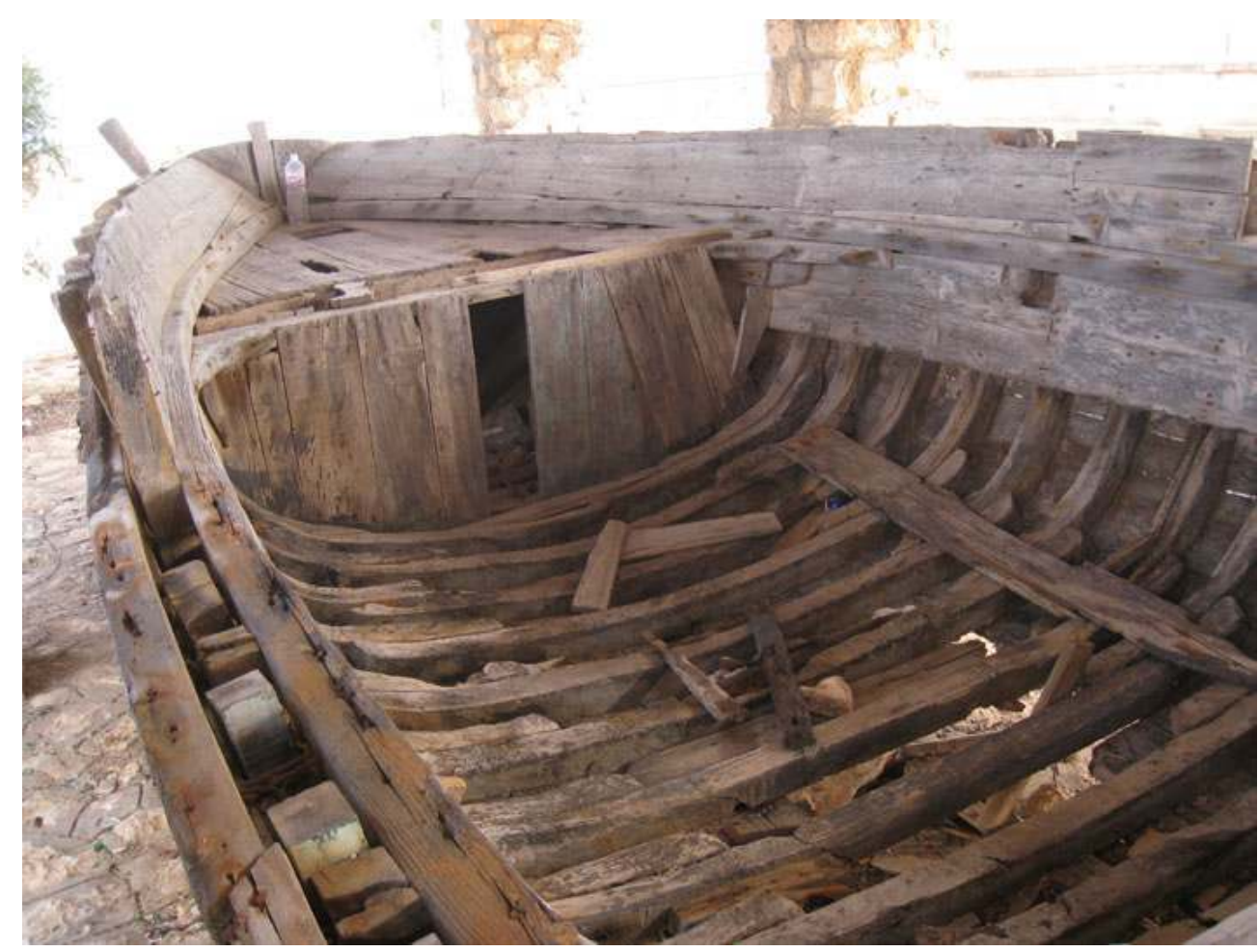

On voit le fond plat du loude vers l'arrière

P. Feron 2014. 


\section{Loude d'el Khraïb, Kerkenna.}

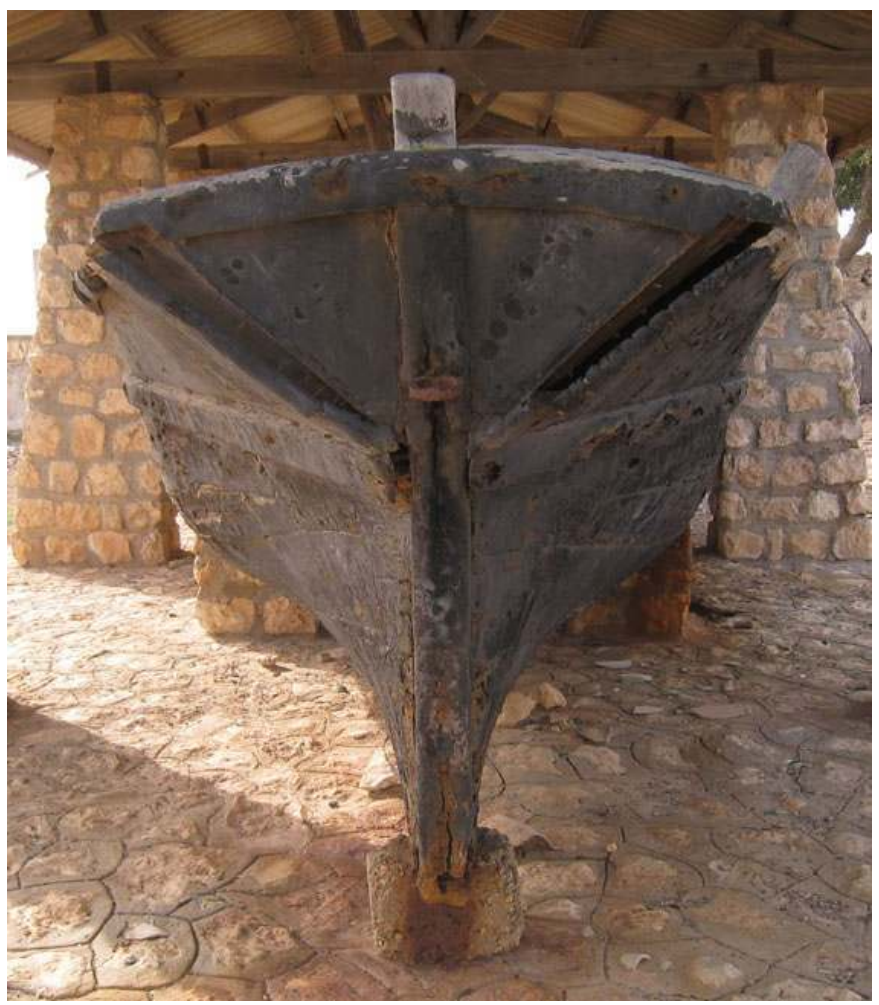

Les lignes d'eau se resserrent en forme de V, sur l'étambot comme sur l'étrave. P. Feron 2014

\section{Loude d'el Khraïb, Kerkenna}

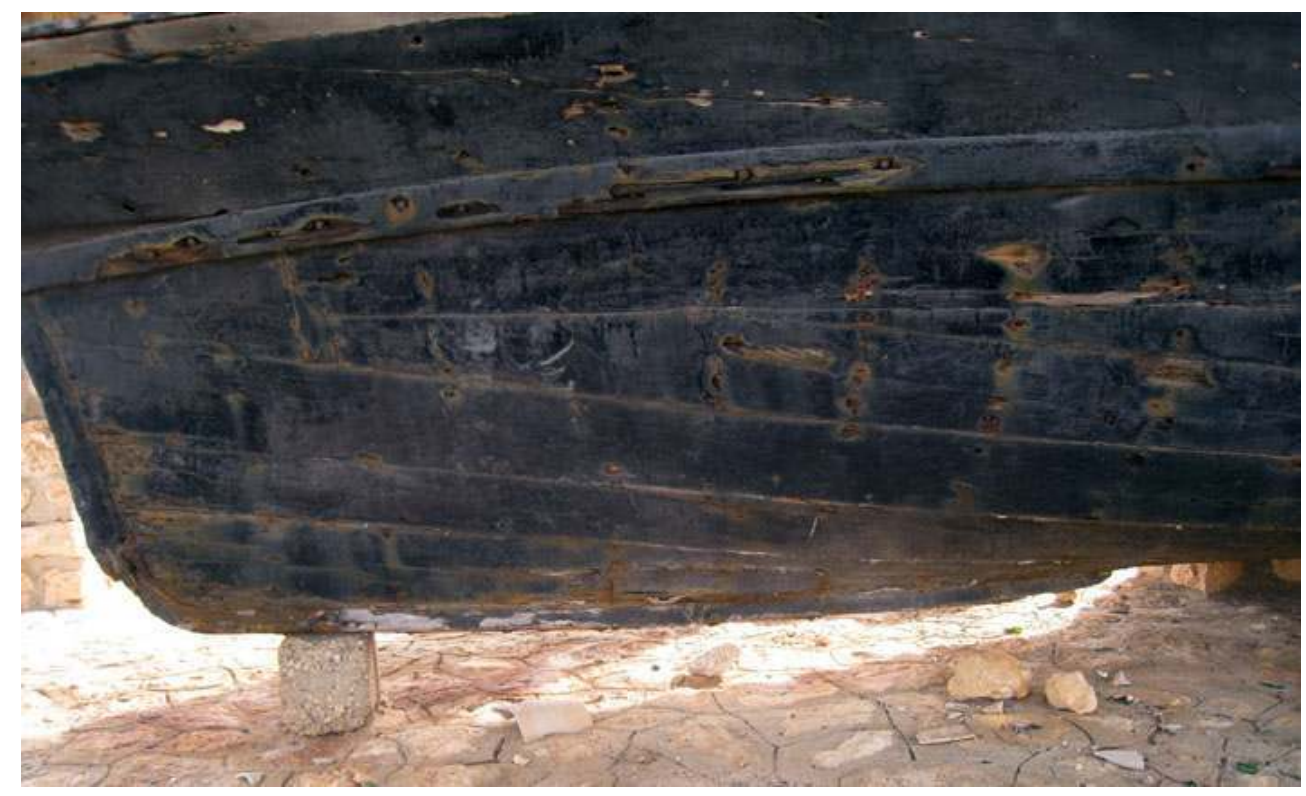

L'embryon de quille du porte l'ossature et le bordage des extrémités en forme de V. P. Feron 2014 
Loude d'el Khraïb, Kerkenna

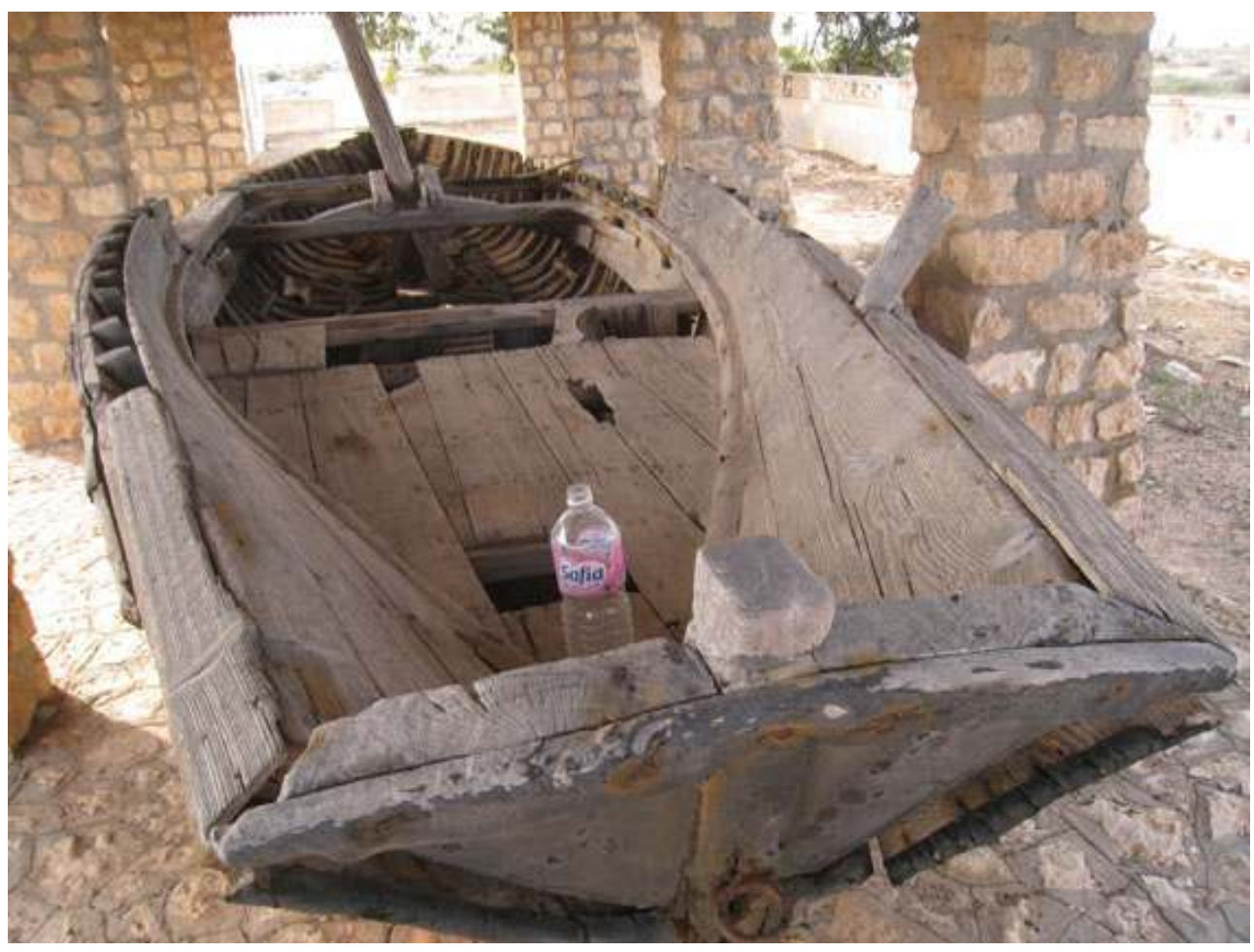

Les fargues qui exhaussent le bordage sur la partie arrière se raccordent au tableau triangulaire. P. Feron 2014 


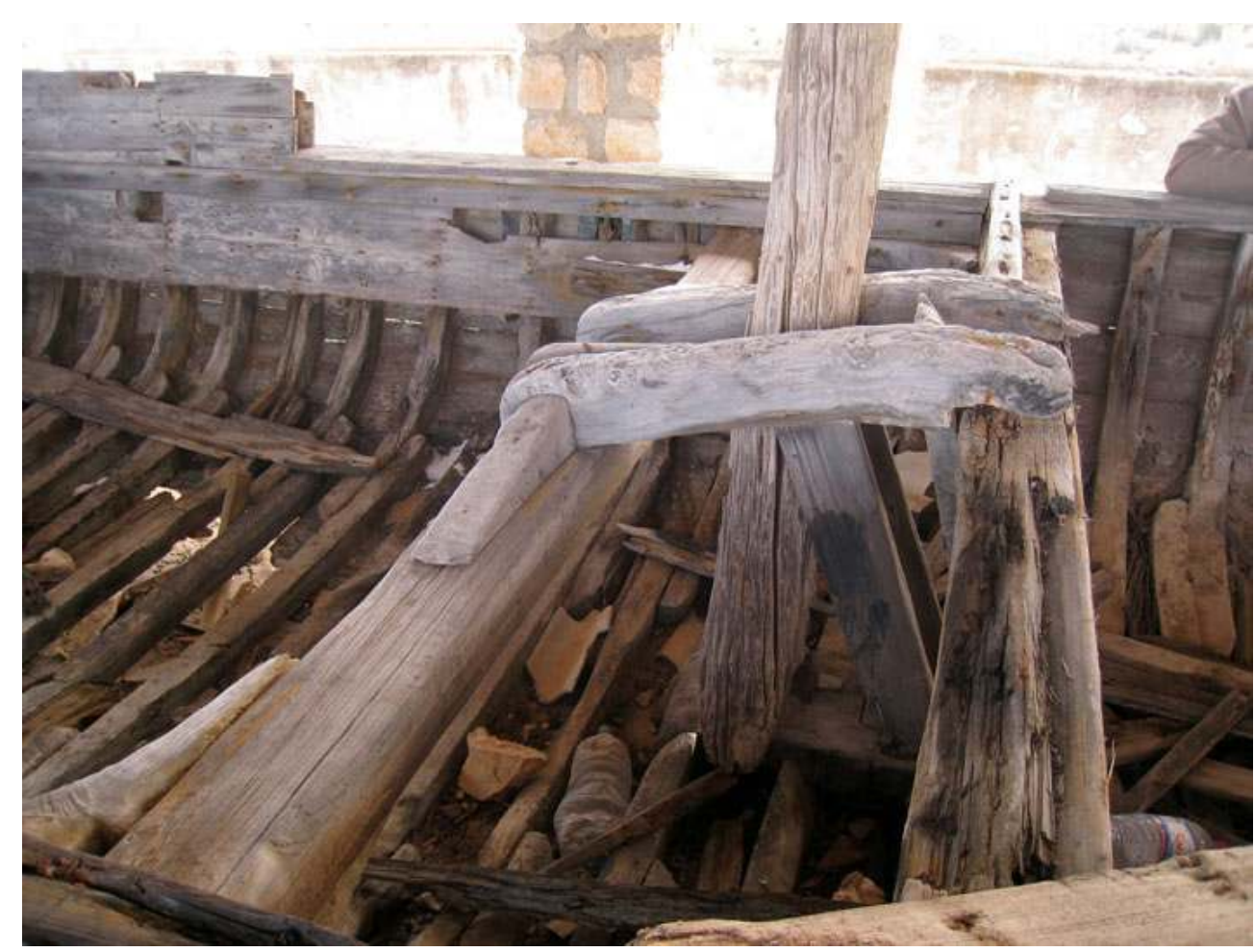

Le dispositif d'étambrai du grand mât doit être très robuste. L'écartement entre les deux barrots transversaux permet l'inclinaison du mât. Les deux entremises, fixées sur les barrots, maintiennent le pied du mât latéralement.

P. Feron 2014

Les éléments d'information relevés dans les textes complètent ceux que fournit le loude d'el-Khraïb. Ils suggèrent un réglage dynamique de son gréement facilitant la manœuvrabilité. La longueur de la coque du loude oscille de 9 mètres à 12 mètres, communément elle mesure 11 mètres, sa largeur au maître bau est de 2,60 mètres. Le creux varie entre 0,80 mètre et 1,00 mètre, son tirant d'eau est de 0,50 mètre, sa capacité moyenne est de 5 tonneaux. Le grand mât peut s'incliner sur l'arrière jusqu'à former un angle mesurable par rapport à l'axe vertical. Sur les photographies ${ }^{35}$, on relève une quête arrière qui varie entre $20^{\circ}$ et $30^{\circ}$, voire $40^{\circ}$ sur les remarquables croquis réalisés par Hennique (fig.12, 13). Le grand mât est soutenu par un étai équipé d'un palan frappé sur l'étrave, et par un hauban de chaque bord. Ainsi, le plan de la grand voile peut osciller d'avant en arrière. Le réglage permet de déplacer son centre vélique par rapport au centre de carène qui est fixe. La manœuvre du plan de voilure d'une planche à voile par rapport à l'axe du vent s'offre en exemple. Quand le vent touche la voile par le travers arrière, le mât est poussé vers l'avant. À l'allure du près, le mât est basculé vers l'arrière, ce qui améliore la remontée au vent. La voile à bourcet ou au tiers, de forme quadrangulaire envoyée sur un mât assez court facile à tenir, offre une grande surface et une voile qui s'oriente très bien au plus-près serré, nous dit le dictionnaire de marine à voile Bonnefoux ${ }^{36}$. Pour un voilier non motorisé l'allure du plus-prés serré est celle qui convient à la pêche à la foëne au même titre que la prise d'une bouée de nasse ou pour mouiller l'ancre. 
Loude au mouillage.

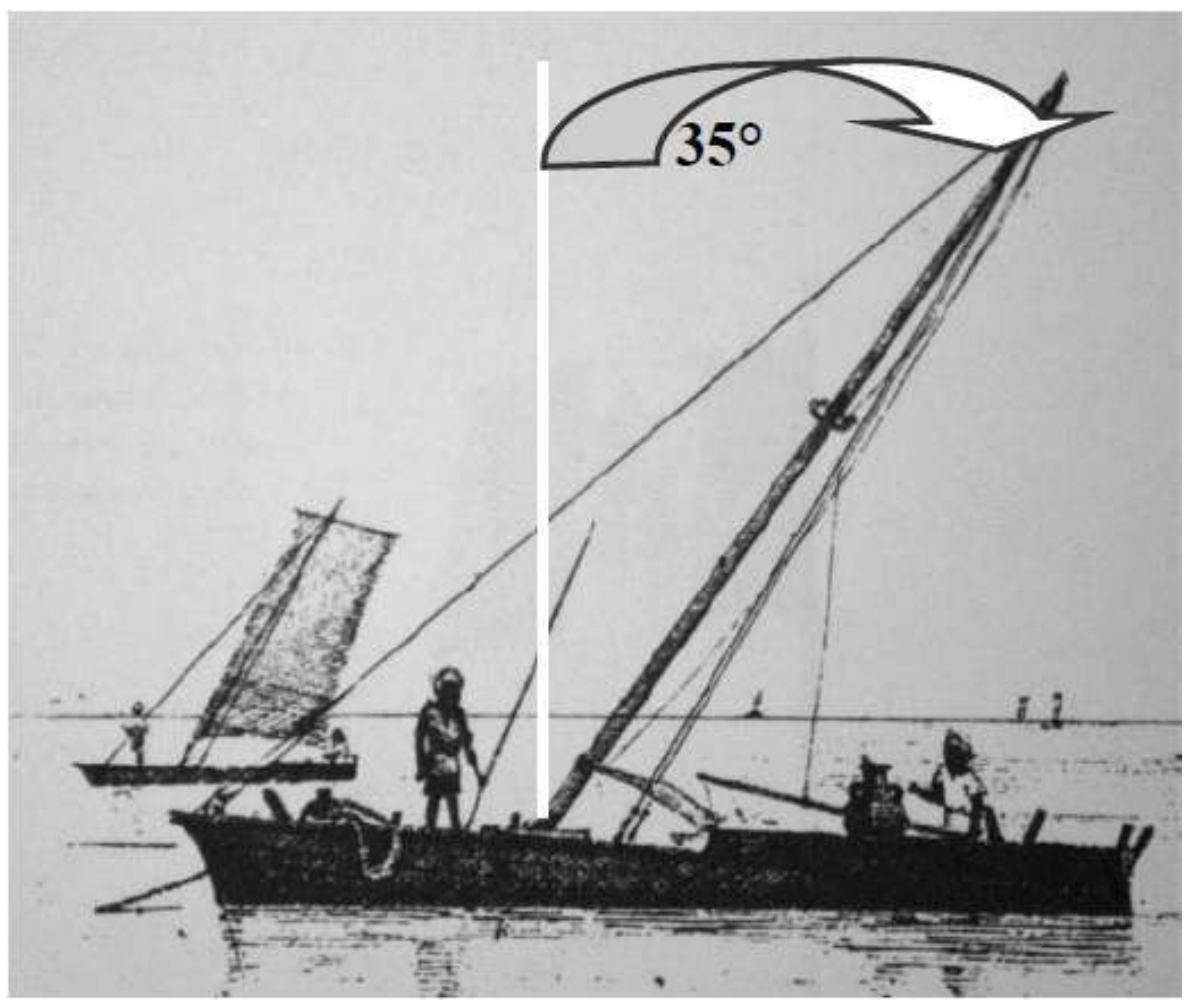

Son mât est incliné à $35^{\circ}$ vers l'arrière. Le pêcheur est debout sur le pont avant, il tient une foëne. Le barreur est assis à l'arrière. À l'arrière-plan, un loude, en action de pêche, navigue sous grand voile. Infographie P. Feron 2014, d'après Hennique, 1884. 


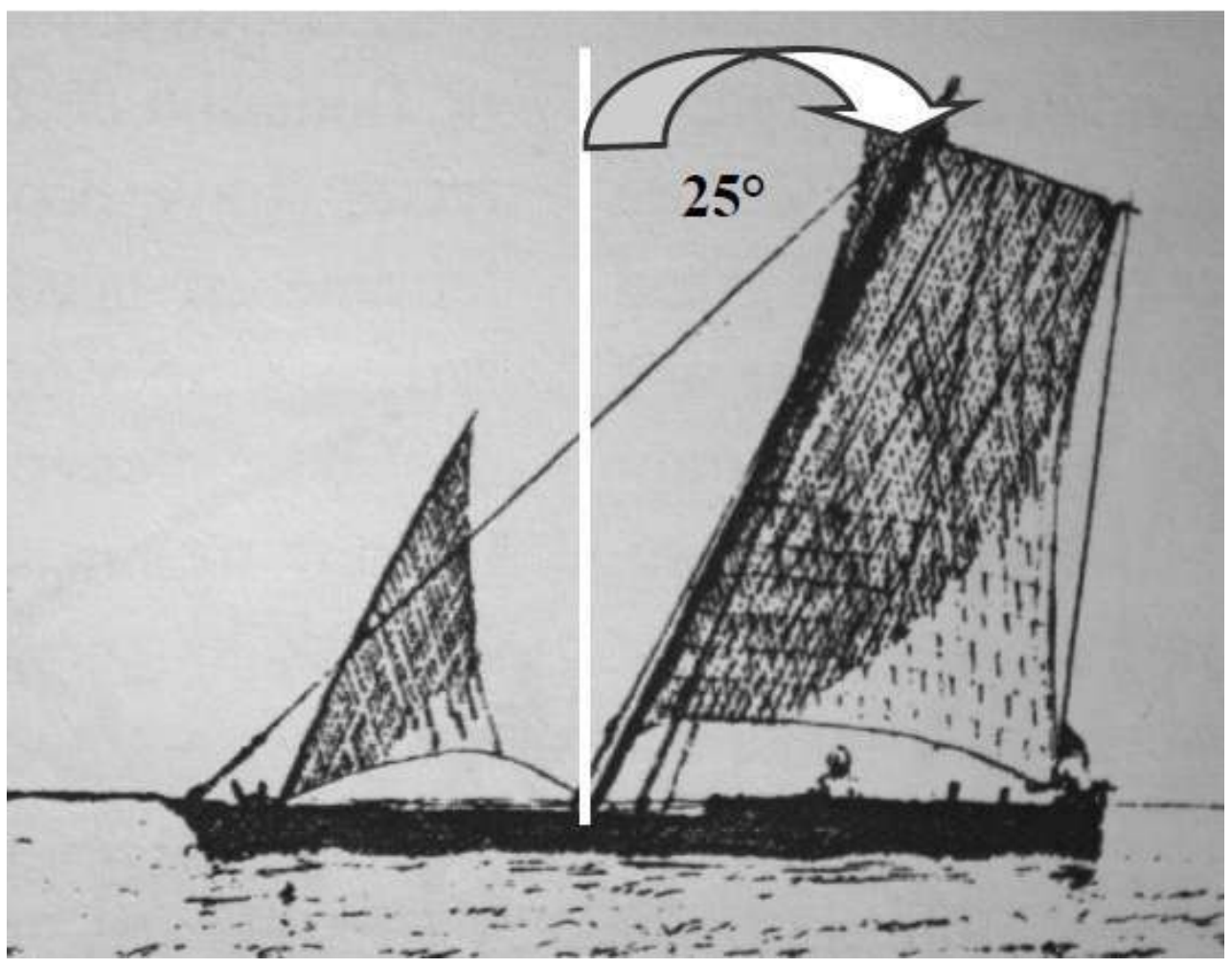

Le mât principal est incliné à $25^{\circ}$ vers l'arrière.

Infographie P. Feron 2014, d'après Hennique, 1882.

On relève chez Hennique ${ }^{37}$ une configuration de gréement qui augmente la surface de voilure et confère plus de puissance. À la voile du grand mât s'ajoute une voile triangulaire ou «trinquet ». Elle est enverguée sur un petit mât placé à l'avant, un espar léger et souple qui fléchit sous la pression du vent. Le centre vélique moyen se déplace sur l'avant et procure un meilleur équilibre par vent portant (fig.14). Ainsi équipée, la silhouette du loude confirme sa filiation avec la famille du carèbe tunisien. À l'époque d'Hennique, le loude est le bateau favori des pêcheurs tunisiens tant pour relever les nasses des pêcheries fixes que pour la cueillette des éponges. La technique de pêche « en route » se pratique sous grand-voile seule, à deux équipiers. Le patron à la barre contrôle le cap et conserve l'allure du près-serré. Le pêcheur se tient debout sur la plage avant, armé d'une longue foëne, il scrute le fond d'une profondeur variable pendant que la barque avance lentement. Dès qu'il aperçoit le refuge d'un poisson dans les herbes ou une éponge, il plante sa fourche dans le fond en guise d'ancre. Le barreur brise l'erre du bateau en venant face au vent. Ensuite, il vient en aide au pêcheur qui capture le poisson ou cueille l'éponge à l'aide d'une seconde foëne. Les poulpes se prennent de façon identique. À travers cette technique de pêche, l'ergonomie du pont avant, les qualités véliques du loude, le savoir-faire nautique des pêcheurs sont pleinement révélés ${ }^{38}$. Selon Hennique, la pêche sur le plateau des Kerkenna est pratiquée presque exclusivement par des loudes, il en dénombre environ 200 unités, puis 75 à Sfax et 100 à Djerba. Le total de l'inventaire s'élève à 454 unités. De Bizerte à Bibène, au sud de Djerba, le loude représente alors $56 \%$ de la flottille de voiliers (fig.15). Désormais, la fluka ${ }^{39}$ des îles Kerkenna occupe l'espace historique du loude. Ce dernier considéré à fond plat, pêchait sur les hauts fonds 
du pré carré poissonneux, il naviguait entre Sfax et l'archipel et plus au large démontrant sa polyvalence lagunaire et maritime.

Allures, way of sailing.

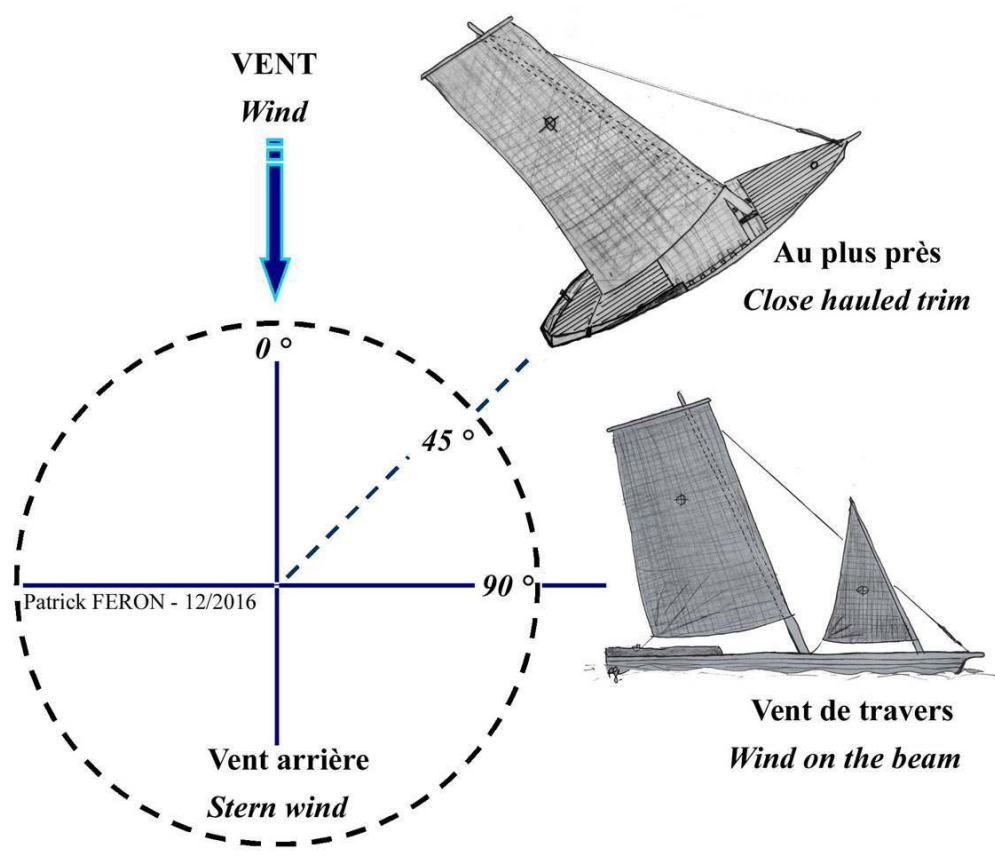

ALLURES, Ways of sailing

Infographie, P. Feron, 2016

Répartition des bateaux à voile sur la côte de Tunisie en 1882 et pourcentage de représentativité.

\begin{tabular}{|c|c|c|c|c|c|c|c|c|c|c|c|c|c|}
\hline & Bricks & \begin{tabular}{|l|} 
Scounas \\
\end{tabular} & Chabêks & Chitihas & $\begin{array}{l}\text { Tartanes } \\
\& \text { cotres }\end{array}$ & Chifazzi & \begin{tabular}{|l|} 
Lanchas \\
\end{tabular} & \begin{tabular}{|l|} 
Misticos \\
\end{tabular} & \begin{tabular}{|l|} 
Sandales \\
\end{tabular} & Speronaras & Carébes & Loudes & Total \\
\hline $\begin{array}{l}\text { Bizerte, Porto-Farina, La } \\
\text { Goulette et Tunis }\end{array}$ & & & & & & & 68 & & & & & & 68 \\
\hline $\begin{array}{l}\text { Kalibia Hammamet, } \\
\text { Souse, Monastir, } \\
\text { Méhédiah, Shebba }\end{array}$ & 1 & 3 & 1 & & 6 & 2 & 17 & & & 2 & 1 & 40 & 73 \\
\hline $\begin{array}{l}\text { Du cap Kadijah à Sfax \& } \\
\text { Iles Kerkennah }\end{array}$ & 6 & 7 & 9 & 3 & 4 & 3 & 19 & 12 & & 2 & 62 & 300 & 427 \\
\hline $\begin{array}{l}\text { Maharess, Surkenis, } \\
\text { Gabes, Ile Djerba, Zarzis }\end{array}$ & 5 & 15 & 16 & 4 & & 3 & 5 & 4 & 34 & 4 & 38 & 114 & 242 \\
\hline Total par type & 12 & 25 & 26 & 7 & 10 & 8 & 109 & 16 & 34 & 8 & 101 & 454 & 810 \\
\hline Représentativité & $1,48 \%$ & $3,86 \%$ & $1,89 \%$ & \begin{tabular}{|l|l|}
$0,86 \%$ \\
\end{tabular} & $1,23 \%$ & $0,58 \%$ & $13,45 \%$ & $1,97 \%$ & $4,19 \%$ & $0,98 \%$ & $12,46 \%$ & $56 \%$ & \\
\hline
\end{tabular}

Infographie P. Feron 2014, d'après Hennique 1884

Dans le sillage de la fluka dont l'usage s'impose sur celui du loude, Louis pointe le moment d'effacement déterminant d'un pan de traditions d'architecture nautique: "Après la disparition des carèbes ou gros loudes, que nous avons vu à l'abandon durant de nombreuses années dans le port de Kellabine, c'est maintenant le tour des loudes ordinaires de disparaître » précisant que les bateaux à fond plat de plus fort tonnage ne sont plus nécessaires. L'approvisionnement en sparte et en alfa, fibres végétales consommées par l'artisanat, se fait à Sfax, aussi le transport depuis Gabès ou Zouara par la mer n'est plus justifié ${ }^{40}$. L'épisode est révélateur de la mouvance temporelle de traditions d'architecture nautique en voie d'obsolescence au sein d'une société insulaire, elle-même en évolution. Le recul de soixante-dix d'années dont nous disposons grâce au travail d'André Louis, nous permet 
d'identifier une mutation qui s'exprime par l'usage croissant et massif d'une barque héritière de la tradition d'architecture nautique sur quille. La flottille de loudes des Kerkenna était encore estimée à 200 unités et 50 à Sfax en $1882^{41}$. La construction du loude était l'apanage de quelques familles kerkeniennes de Chergui dont la compétence était largement reconnue. L'une d'elle tenait un chantier à Sfax. Le secret des charpentiers résidait tout simplement dans un savoir-faire vernaculaire et le prêt de gabarits entre membres de la famille. Mais déjà, le loude ne se construisait presque plus ${ }^{42}$. En 1966, les trois derniers exemplaires observés en baie d'Ech-Chergui sonnent le chant du cygne du loude à Kerkenna ${ }^{43}$. En 1981, le dernier représentant de la famille de charpentiers ne construisait plus que des maquettes de loudes destinées aux touristes.

Cette rupture introduit un principe de construction tributaire d'un tirant d'eau plus fort à priori, cependant au prix d'un métissage des formes qui aplatit le fond et malgré la saillie de sa quille, la fluka se satisfait de très peu d'eau pour flotter. Le bassin nautique investi par les insulaires se concentre désormais dans le premier cercle, celui des hauts fonds et des chenaux intermédiaires poissonneux où la fluka sert à rejoindre les pêcheries fixes.

Quand Éric Rieth interroge les «deux territoires socioculturels de Sfax de 1939 et des Kerkenna du début du XXI siècle » il relève une " proche identité de culture technique » chez les charpentiers navals, distinguant néanmoins une évolution du vocabulaire. Ce constat témoigne d'un transfert technique et de la conservation du savoir-faire de la construction sur quille. Concernant l'emploi du maître-gabarit, et les liens éventuels avec le monde arabe, le sujet renvoie au Moyen Âge précise-t-il, illustrant ainsi la profondeur du temps long ${ }^{44}$.

31 "Les Kerkenna disparaitront-elles?» se demandait André Louis. Si l'on constate aujourd'hui que des facteurs de résilience naturels et coutumiers favorisent la conservation de cultures techniques et de traditions nautiques dans l'archipel, si dans ce processus la fluka maintient la mémoire vivante d'une ultime mutation, il est permis de s'interroger sur la fragilité du terme « espace nautique conservateur » et sur sa durabilité. Ce survol spatio-temporel a matérialisé la dilution progressive de la diversité patrimoniale de bateaux vernaculaires qui croisaient dans les eaux du golfe de Gabès et des Kerkenna. Le phénomène qui s'est généralisé sur la majeure partie des façades maritimes, reflète un bouleversement profond de l'économie et de l'industrialisation du monde de la mer, à commencer par la motorisation des bateaux qui concourt à l'abandon de la voile. Dans ce contexte de disparition, deux facteurs récents apparaissent, la dérive des pratiques halieutiques professionnelles actuelles affecte gravement l'équilibre écologique de l'espace maritime qui baigne l'archipel des Kerkenna. Enfin, les relations sociales frappées autrefois du sceau de la culture traditionnelle tendent à se déliter rapidement.

33 Au début de l'année 2015, l'actualité témoignait de la tension qui régnait chez les pêcheurs recourant aux nouvelles techniques de pêche pratiquées avec des barques motorisées. Ces derniers avaient bloqué l'accès des bateaux de transport à Kerkenna afin de s'opposer à des mesures de régulation de la pêche. Contrairement à la gestion raisonnable des anciens opérateurs, les techniques nouvelles, le non respect des usages sociaux et de la réglementation en vigueur détériorent la flore et la faune. À la surexploitation s'ajoute le non respect des cycles biologiques d'espèces variées, des périodes et des zones de pêche autorisées, aussi le secteur de la pêche à Kerkenna est-il dans l'incertitude. 

maritime est remis en cause. L'empilement de pratiques de pêche, telle que la pose de filets invisibles dans les petits fonds proches des pêcheries, se superposant aux pierres creuses et aux gargoulettes qui servent à la capture des poulpes, provoquent une perturbation des activités de la pêche coutumière. Le Kiss, ou filet invisible petit modèle est apparu en 1960 ; malgré l'interdiction du législateur, le grand Kiss, s'est imposé en 1975. Pour la conduite de l'exploitation halieutique traditionnelle les kerkenniens utilisaient des barques à rames et à voiles. Accompagnant l'intrusion de nouveaux procédés de capture, la motorisation des barques fut encouragée par des prêts. Le chevauchement déraisonnable et incontrôlé de ces différents modes de pêche parfois inadaptés au milieu, associé à la surexploitation croissante du stock par augmentation du nombre de barques et de la puissance moteur, dégradent l'équilibre biologique du milieu, menaçant l'économie des techniques traditionnelles ${ }^{45}$.

\section{Conclusion}

L'anneau de hauts fonds prolifiques qui ceinturent les îles Kerkenna tel un "pré carré poissonneux ", constitue jusqu'à ce jour le principal ressort conservateur d'une technique de prélèvement ancestrale. Au centre d'un dispositif de pêcheries fixes, le palmier est le pourvoyeur d'un système de capture très ingénieux, grand consommateur de palmes fichées sur les hauts fonds. L'arbre prodigue, facteur végétal de conservation, pérennise la pêcherie fixe depuis des siècles et matérialise le triptyque «Hauts fonds, Palmier \& Savoir-faire ». Aujourd'hui, la fluka, modèle fonctionnel métissé, intervient en parfaite congruence avec le milieu, remplaçant le loude. Elle sillonne avec aisance le territoire halieutique, contribuant à l'exploitation du vaste plateau à fleur d'eau qui porte l'archipel. Elle est aujourd'hui le marqueur d'une tradition d'architecture nautique sur quille, objet symboliquement réfractaire à l'industrialisation des techniques de pêche mais elle tend à se motoriser.

Louis aborde les Kerkenna pour la première fois en 1945, son étude offre un recul de soixante-dix ans, elle nous introduit au cœur même de l'archipel où le radeau de tronc de palmiers appelé etchem, témoin de la tradition dite première, n'est plus qu'une image fugitive fixée en 1925. L'auteur navigue à bord d'un loude, représentant d'une importante flottille de bateaux à fond plat, on n'en comptera plus que trois en 1966. Rétrospectivement, on assiste à l'extinction d'une grande diversité de modèles de bateaux opérant dans le golfe de Gabès et en Méditerranée orientale. Dans leur sillage qui s'efface, la construction de la felouque ou fluka, se développe d'abord dans plusieurs villages de la côte orientale des Kerkenna. La flottille particulièrement représentative que l'on observe aujourd'hui occupe le territoire du loude. Elle brandit le flambeau tunisien de la mouvance de traditions d'architecture nautique au cœur de l'archipel des îles Kerkenna, un microcosme porteur et témoin majeur d'une culture maritime exceptionnelle. Mais, à la question posée par André Louis: "Les Kerkenna disparaîtront-elles?", la pêche intensive et désordonnée qui s'amplifie en défiant le sens coutumier, semble vouloir offrir une réponse positive. 


\section{BIBLIOGRAPHIE}

BARKAOUI Abdelhamid, RIETH Éric (dir), Histoire et mémoire de la construction navale vernaculaire aux îles Kerkenna, Tunisie, Med Ali Editions, Tunis, 2014

BARKAOUI Abdelhamid, RIETH Éric (dir.), Bateaux et ports méditerranéens, Bilan et perspectives, Actes de la Table Ronde internationale, Université de Sfax, Facultés des Lettres et Sciences Humaines de Sfax, Med Ali Editions, Sfax, 2006

BASCH Lucien, « De l'apport de l'iconographie à l'archéologie », in BARKAOUI Abdelhamid, RIETH Éric, (dir.), Bateaux et ports méditerranéens, Bilan et perspectives, Actes de la Table Ronde internationale, Université de Sfax, Facultés des Lettres et Sciences Humaines de Sfax, Med Ali éditions, Sfax, 2006, p.11-27

BONNEFOUX Pierre-Marie-Joseph, PÂRIS Edmond, Dictionnaire de marine à voile, (1847), Éditions du Layeur, Paris, 1999

BRAUDEL Fernand, La Méditerranée et le monde méditerranéen à l'époque de Philippe II. 1. La part du

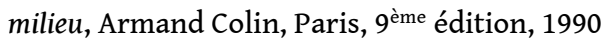

FERHI Abdelhamid, Kerkenna, charme insulaire, Centre Cercina pour les Recherches sur les îles méditerranéennes, Musée-résidence, Kerkenna, Série Rives Méditerranéennes, nXII, 2014

GATEAU Albert, Introduction à l'étude du vocabulaire maritime en Tunisie : Technologie du lûd, Revue Africaine, t.90, 1946, p.140-183

HATTOUR Mohamed Jamel, SAMMARI Chérif, BEN NASSRALLAH Sassi, « Hydrodynamique du golfe de Gabès déduite à partir des observations de courants et de niveaux ", Revue Paralia, vol. 3, 2010, p.1

HENNIQUE Privat-Agathon, « Caboteurs et pêcheurs de la côte de Tunisie en 1882 », Revue Maritime et Coloniale, t. 82, Berger-Levrault, Paris, 1884, p.5-67

HENNIQUE Privat-Agathon, Une page d'archéologie navale, Caboteurs et pêcheurs de la côte de Tunisie, Pêche des éponges, Gauthier-Villars, Paris, 1888

LANIER Lucien, L'Afrique, choix de lectures de géographies, accompagnés de résumés, d'analyses, de notes explicatives et bibliographiques, Belin, Paris, 1884

LOUIS André, Les îles Kerkena, (Tunisie), étude d'ethnographie tunisienne et de géographie humaine, Publications de l'institut des Belles lettres Arabes, Bascone \& Muscat, Tunis, 1961

MAB, Projet 7, Établissements humains et environnement socioculturel, Études interdisciplinaires de l'habitat humain dans les Écosystèmes insulaires, UNESCO, Paris, 1981

RHOUMA Abdelmajid, LABIDI Abdelmajid, Rapport diagnostic participatif de l'état de la pêche traditionnelle aux îles Kerkenna, PNUD, Fonds pour l'Environnement National, Lyons club Sfax/ Thyna, novembre 2006

SERVONNET Jean, LAFFITE Fernand, En Tunisie, le Golfe de Gabès en 1888, réédition, Ecosud, Gabès, 2000 


\section{ANNEXES}

Enquêtes, entretiens, prospections

Enquête et observation ethnographique : le loude d'el-Khraïb-Beït-en-Najet, conservé sous abri, Kerkenna, décembre 2014.

Entretien avec le professeur A. Barkaoui, Faculté des Lettres et Sciences Humaines de Sfax décembre 2014.

Entretien avec le capitaine d'armement de la compagnie de navigation tunisienne, SONATRAK qui dessert quotidiennement l'archipel des Kerkenna, consultation de la documentation nautique de cette zone maritime, Sfax, décembre 2014.

Enquête ethnographique, Chantier Sta Zohir Chelli et entretien, Kerkenna, décembre 2014.

Prospections sur le terrain, visite commentée du Musée, avec le professeur A. Ferhi, Kerkenna, décembre 2014.

\section{NOTES}

1. LOUIS André, Les îles Kerkena, (Tunisie), Tunis, 1961, p. 6. André Louis, membre de l'Institut des Belles Lettres Arabes de Tunis est l'auteur d'une thèse principale pour le doctorat ès Lettres, présentée à la Faculté des Lettres et des Sciences Humaines de l'Université de Paris, elle a été éditée en 1961. L'auteur fait connaissance avec Kerkenna dès 1945 et termine ses travaux de recherche en mars 1956. Son étude d'ensemble comprend trois volumes, deux sont consacrés aux îles, sous l'angle de l'ethnographie et de la géographie humaine. Le troisième documente l'ethnographie et la linguistique sur les îles. Ce travail, réalisé au moment où de profonds changements de société s'amorcent, fait aujourd'hui référence.

2. SERVONNET Jean, LAFFITE Fernand, En Tunisie, le Golfe de Gabès en 1888, Gabès 2000, p.46.

3. BRAUDEL Fernand, La Méditerranée et le monde méditerranéen à l'époque de Philippe II. 1. La part du milieu, Paris, 1990, p.126.

4. BARKAHOUI Abdelhamid, "Le chantier naval traditionnel aux îles Kerkenna (Tunisie) », $3^{e}$ Workshop International, Master Erasmus Mundus TPTI, Université Paris 1 Panthéon-Sorbonne, 8 juillet 2011.

5. Chausey (France), Minquiers, Écréhou, Jersey, Guernesey, Serck, Herm, Aurigny (Îles anglonormandes).

6. BARKAHOUI Abdelhamid, RIETH Éric, Histoire et mémoire de la construction navale vernaculaire aux îles Kerkenna, Tunisie, Tunis, 2014, p.8.

7. André Louis fait référence à André Leroi-Gourhan, « dont les travaux toujours si proche des problèmes humains [1']ont encouragé dans des recherches parfois ardues » et selon lequel "l'enquête linguistique et l'observation des techniques sont deux moyens d'accès privilégiés à l'intelligence des cultures ».

8. Entretien avec le Professeur Barkaoui, Faculté des Lettres et des Sciences Humaines de Sfax, décembre 2014.

9. BARKAHOUI Abdelhamid, Le bateau de l'Africa, Éd. Reliure d'Art, Sfax, 2012; La Marine carthaginoise, Éd. L'Or du Temps, Tunis, 2003. 
10. BASCH Lucien, « De l'apport de l'iconographie à l'archéologie », in BARKAHOUI Abdelhamid, RIETH Éric, (dir.), Bateaux et ports méditerranéens, Bilan et perspectives, Actes de la Table Ronde internationale, Université de Sfax, Facultés des Lettres et Sciences Humaines de Sfax, Med Ali éditions, Sfax, 2006, p. 11-27.

11. BARKAHOUI Abdelhamid, RIETH Éric, «Pour un répertoire de l'iconographie navale du Maghreb, La Tunisie ", in Bateaux et ports méditerranéens, Bilan et perspectives. Actes de la table ronde internationale, Sfax-Kerkenna, 6-7 avril 2005, Éd. Medi Ali, Sfax, 2006, p. 29-63.

12. Enquête ethnographique au chantier Sta Zohir Chelli et entretien, Kerkenna, décembre 2014.

13. BARKAHOUI Abdelhamid, Notes sur le voilier tunisien ou une tradition nautique en voie d'extinction, le cas du loud d'el Khraib-Beït-en-Najet, (2002-2003), Faculté des Lettres et des Sciences Humaines de Sfax, s. d. p. 41-58.

14. SERVONNET Jean, LAFFITE Fernand, En Tunisie... op.cit. 2000.

15. HENNIQUE Privat-Agathon, "Caboteurs et pêcheurs de la côte de Tunisie en 1882 ", Revue Maritime et Coloniale, t. 82, Berger-Levrault, Paris, 1884, p. 5-67; Une page d'archéologie navale, Caboteurs et pêcheurs de la côte de Tunisie, Pêche des éponges, Gauthier-Villars, Paris, 1888.

16. GATEAU Albert, «Introduction à l'étude du vocabulaire maritime en Tunisie:Technologie du lûd », Revue Africaine, t.90, 1946, p.140-183.

17. BRAUDEL Fernand, La Méditerranée et le monde méditerranéen à l'époque de Philippe II. 1. La part du milieu, Paris, 1990, p.126-127.

18. Entretien avec le capitaine d'armement de la compagnie de navigation tunisienne SONATRAK. Elle dessert quotidiennement l'archipel des Kerkenna, consultation de documents nautiques, Sfax, décembre 2014.

19. HATTOUR Mohamed Jamel, SAMMARI Chérif, BEN NASSRALLAH Sassi, « Hydrodynamique du golfe de Gabès déduite à partir des observations de courants et de niveaux ", Revue Paralia, vol. 3, 2010, p.1.

20. Ibidem, p.2-3, 4 .

21. MAB-Projet 7, Établissements humains et environnement socioculturel, Études interdisciplinaires de l'habitat humain dans les Écosystèmes insulaires, Paris, 1981, p.80.

22. LOUIS André, Les îles Kerkena..., op.cit., 1961, p.170.

23. La transformation du sparte en cordage est devenue aujourd'hui obsolète, cette graminée originaire du Sahel alimentait un artisanat diversifié intense et très productif, le loude participait à son transport vers Kerkenna.

24. LOUIS André, Les îles Kerkena..., op.cit., 1961, p.220. L'étude de M. A. Louis s'arrête en février 1956.

25. FERHI Abdelhamid, Kerkenna, charme insulaire, Kerkenna, 2014, p.45.

26. La perche est une gaffe plus longue.

27. LOUIS André, Les îles Kerkena..., op.cit., 1961, p.72, 80, 108-109, 213.

28. Albert Gateau, cité par André Louis, ibidem, p. 214, 215.

29. Introduit en Algérie en 1861, la culture de l'eucalyptus globalus se développa à partir de 1884 . LANIER Lucien, L'Afrique, choix de lectures de géographies, accompagnés de résumés, d'analyses, de notes explicatives et bibliographiques, Paris, 1884, p.142.

30. HENNIQUE Privat-Agathon, « Caboteurs et pêcheurs... », op.cit., 1884, p.12-14, 17, 19.

31. Observation ethnographique du loud d'el Khraïb-Beït-en-Najet, Kerkennah, décembre 2014 ; Barkaoui, 2003, « Note sur le voilier tunisien ou une tradition nautique en voie d'extinction, le cas du loud d'el-Khraïb-Beït-en-Najet à Kerkenna », dans Les méditerranéennes, Relais de civilisations, Série Rives méditerranéennes, Livre IV, sept. 2004, p. 41-48. (Enquête ethnographique, 2002-2003) 32. L'expression «Barque à fond plat, adaptée à la navigation sur les hauts fonds ", à propos du loude, est employée par André Louis en se référant à Albert Gateau.

33. HENNIQUE Privat-Agathon, Une page d'archéologie navale, Caboteurs et pêcheurs de la côte de Tunisie, Pêche des éponges, Paris, 1888, p. 20. 
34. André Louis remarque la répétition du même geste soixante ans après la relation d'Hennique.

35. LOUIS André, Les îles Kerkena..., op.cit., 1961, p.214-215, fig. 79, 80.

36. BONNEFOUX Pierre-Marie-Joseph, PÂRIS Edmond, Dictionnaire de marine à voile, (1847), Paris, 1999 , p.111.

37. HENNIQUE Privat-Agathon, Une page..., op.cit., 1888, p.20, 22, 64.

38. Dans cette configuration, le loude s'intègre dans un système technique spécifique à la pêche à la voile.

39. On évalue approximativement cette flottille à plus de 2000 unités, la moitié des armateurs sont des pêcheurs professionnels, décembre 2014.

40. Le sparte ou faux alfa est une graminée qui provient du sud du Sahel. Il est roui dans l'eau de mer, puis toronné en cordelettes, cordes et cordages, ou tressé en nattes et couvre-bât de chameaux. L'alfa et le sparte se combinent dans la confection d'engins de pêche. LOUIS André, Les îles Kerkena..., op.cit., 1961, p.219, 339.

41. HENNIQUE Privat-Agathon, « Caboteurs et pêcheurs... », op.cit., 1884, p.63.

42. LOUIS André, Les îles Kerkena..., op.cit., 1961, p.247.

43. MAB-Projet 7, Établissements humains..., op.cit., Paris, 1981, p.105.

44. BARKAOUI Abdelhamid, RIETH Éric, Histoire et mémoire..., op.cit., 2014, p.69 et 90.

45. RHOUMA Abdelmajid, LABIDI Abdelmajid, Rapport diagnostic participatif de l'état de la pêche traditionnelle aux îles Kerkenna, Sfax, 2006, p.3, 21 et 30.

\section{RÉSUMÉS}

Le loude d'el-Khraïb-Beït-en-Najet à Kerkennah est le dernier témoin d'une flottille de voiliers tunisiens aujourd'hui disparue. Son principe de construction à fond plat et sa manœuvre sous voile correspondaient parfaitement à la pratique de la pêche sur les hauts fonds. Au XX ${ }^{\mathrm{e}}$ siècle, la construction de la fluka s'est développée dans plusieurs villages de la côte orientale des Kerkennah. Cette barque construite sur quille occupe aujourd'hui le territoire historique du loude. L'étude explore l'évolution récente de ces deux bateaux traditionnels, ultimes représentants culturels de l'histoire maritime des Kerkennah.

The «loud»" of el-Khraib-Beit-en-Najet, at Kerkennah is the last witness of a flotilla of Tunisians sailboats, which does not exist anymore. Its flat-bottomed hull and its sail shape perfectly matched the practice of fishing on shoals. However, in the twentieth century, flukas were built in several villages on the eastern coast of Kerkennah islands. This boat built on keel today occupies the historic territory of the Loud. This study explores the recent evolution of these two traditional boats, which are the last cultural representatives of the Kerkennah's maritime history.

\section{INDEX}

Mots-clés : histoire des techniques, Tunisie, Îles Kerkennah, XIXe-XXIe siècles, espace nautique, loude, fluka

Keywords : history of technology, Tunisia, Kerkennah Islands, XIXth-XXIth century, nautical area, loude, fluka 


\section{AUTEUR}

\section{PATRICK FÉRON}

Consultant maritime et fluvial, Patrick FERON achève une thèse sur le métissage de la tradition d'architecture nautique européenne en Afrique de l'ouest et centrale entre le XIX et le XX $\mathrm{XX}^{\mathrm{e}}$ siècle dans le cadre du programme doctoral international HERITECHS, à l'Université Paris 1 PanthéonSorbonne. Ses recherches portent plus généralement sur le développement de traditions d'architecture nautiques vernaculaires. 